\title{
Cloud based Financial Market Prediction through Genetic Algorithms: A Review
}

\author{
Nitasha Soni \\ Research Scholar, Lingaya's University \\ Nachauli, Jasana Road, Old Faridabad, \\ Nacholi, Haryana 121002
}

\author{
Tapas Kumar, PhD \\ Professor, Lingaya's University, \\ Nachauli, Jasana Road, Old Faridabad, \\ Nacholi, Haryana 121002
}

\begin{abstract}
This paper surveys recent literature in the area of stock market forecasting using advanced engineering based methods like Neural Network, fractal theory, Data Mining, Hidden Markov Model and Neuro-Fuzzy system. Neural Networks and NeuroFuzzy systems are emerging as an effective tool to be used in the forecasting of stock market especially in machine learning techniques. Due to chaotic behavior of the market, traditional techniques are insufficient to cover all the possible relation of the stock price fluctuations. Neural Network and Markov Model is being used exclusively in the forecasting of finance markets but in third world countries. In this paper, we will discuss the relevance of existing methods based on neural network and discussed gaps between these methods. We also propose a forecasting method to provide better an accuracy rather traditional method.
\end{abstract}

\section{Keywords}

Neural Network, Data Mining, Stock Market Prediction, Markov Model, Neuro-Fuzzy Systems, Forecasting Techniques, and Time Series Analysis.

\section{INTRODUCTION}

Stock market forecasting is an art to predict the market with the reason. A stock market index represents the health of economy. Many tools are used in the forecasting process by professionals but some of the key characteristics of individual stock are not taken into consideration. To overcome this limitation, the researchers could develop a model to forecast individual stock prices. Returns on equity are dependent upon various factors e.g. risk taken, time of entry, exit time, duration of the investments etc. Information plays a key factor in the investment process. Tools used for the prediction of the market are broadly divided into two parts i.e. statistical and non statistical. There is long history of statistical tools used in the stock market. The prominent charts used in statistical methods are i.e Candlestick Charting, Moving Averages, Parabolic SAR, Bollinger Bands, Donchian Channels, Comparative Strength, Gaps, Correlation, Andrew's Pitchfork etc. The major drawback of statistical method is that it doesn't discount all factors of stock market. Alternative methods are emerging as a new robust method which include majority of factors. The survey of recent techniques such as Neural Network, Data Mining, Markov Model and Neuro-Fuzzy system offer useful tools for forecasting noisy environments like stock market. Stock markets are chaotic in nature and it follow fractal market hypothesis. Fractal market hypothesis follows following five rules [6]:

FMH1. The market is made up of many individuals with a large number of different investment horizons.
FMH2. Information has a different impact on different investment horizons.

FMH3. The stability of the market is largely a matter of liquidity (balancing of supply and demand). Liquidity is available when the market is composed of many investors with many different investment horizons.

FMH4. Prices reflect a combination of short-term technical trading and long-term fundamental valuation.

FMH5. If a security has no tie to the economic cycle and then there will be no long-term trend. Trading, liquidity, and shortterm information will dominate.

Fractal market hypothesis points out some more interesting facts and figures e.g it is more concerns about participation of the stock market players and investors, chaotic behavior of the stock market etc. Therefore modern tools for forecasts must consider these before they come to any conclusions. Many researchers using different tools and methodologies while investing in the market. Results of the process and return are dependent upon efficiency of the tool used and personal acumen of the investors. Although we know that effectiveness of the forecasting tool is dependent upon market conditions and all tools are not always effective in all conditions. So, selection of tools and methodologies are very much important. Prediction with database requires new school of thought over database to introduce green computing in stock market with cloud and through new algorithm we can reduce database volume for efficient use $[10,11]$ Over the period of time, neural network has emerged as excellent tool to predict the market. Aim of the research is to critically analyze the alternative tools specially based on the neural networks in the stock market. It also surveys the recent techniques such as Neural Network, Data Mining, Markov Model and NeuroFuzzy system as tools for forecasting stock market.

\section{LITERATURE REVIEW}

Literature is full of methodologies to forecast the market. In spite of all these theories developed by researchers, it's still tough to predict stock market exactly in different conditions of the market. The stock market indices are highly fluctuating and chaotic in nature. Fluctuations are based upon many factors and its affects investor's belief. Searching and deciding most effective method to predict stock market requires ample information about nitty-gritty of the market happing. A stock market index represents the directional movement economy. To forecast the market indices or individual stock, we require more accurate and suitable formulae which help in predictions. In 2010, Prof. R.K. and Pawar D.D. in [4] find out more accurate and effective ways to predict the market. According to them, predictions are 
based upon stock market indices signals. They advocated that artificial neural network may be more suitable for the task. Neural network is suitable to extract large set of useful information. Halbert white [7] discovered some results of an on-going project using neural network modeling and learning techniques to search for and decode nonlinear regularities in asset price movements. Jing Tao Yao and chew Lim tan [8] uses neural networks for classification, prediction and recognition of the patterns of the stock market. He said that trading with neural network is an art. He proved that neural network module is able to predict the market efficiently. Data processing/analysis is accurately done with the help of neural network model. Tiffany Hui-Kuang and Kun-Huang Huarng [16] used neural network for forecasting because it has capability to deal with nonlinear relationship of the stock market. He also introduces new fuzzy time series model to improve forecasting. Tiffany Hui-Kuang and Kun-Huang Huarng uses data from Taiwan stock market to show its results. Akinwale adio T, Arogundade O.T and Adekoya Adebayo F in 2009 [2] examined back propagation and regression analysis to predict the un-translated and translated stock market and uses Nigeria Stock Market Price (NSMP) as data to prove their results. Akinwale adio T, Arogundade O.T and Adekoya Adebayo F used network topology with five input variable in the stock market. Enke and Suraphan Thawornwong [5] advocated machine learning data mining tool to forecast that market. Has had evaluate the predictive relationship of many financial products on various parameters and predict successfully on the same. They proof that future forecasting of stock market can be done after estimation and classification of neural network. A cross-validation technique was used to improve the generalization ability of several models. Yi-Fan Wang, Shihmin Cheng and Mei-Hua Hsu [18] used Markov chain concepts into fuzzy stochastic prediction of stock indexes to achieve better accuracy and confidence. In this paper he compared ANN and Markov model as a major tool for forecasting. It produces more refined result. K. Senthamarai Kannan, P. Sailapathi Sekar, M.Mohamed Sathik and P. Arumugam [9] used data mining technology to discover the hidden patterns from the historic data that have probable predictive capability in their investment decisions. Time series analysis capabilities can be generated by the use of data mining tools. Five Methods namely Typical price (TP), Bollinger bands, Relative strength index (RSI), CMI and moving average (MA) used to analyzed the stock index. In this paper the author got the profitable signal is $84.24 \%$ using Bollinger Bands rather than MA, RSI and CMI Abdulsalam sulaiman olaniyi, adewole, kayoed s, Jimoh R.G [1] used moving average [MA] to deal with patterns of the market behavior from different database to forecast the market. M. suresh Babu, N. Geethanjali and B. Sathyanarayana [13] advocates mining methods to uncover the hidden pattern in stock market. Pattern discovery techniques is descriptive in time series analysis. In this paper, M. suresh Babu, N. Geethanjali and B. Sathyanarayana used new algorithm to deal with dynamic pattern in time series analysis. Youngohc Yoon and George Swales (19) in1991 established neural network as tool to capable of learning function that maps. They compared Neural Network technique with Multivariate Discriminate Analysis approach Indicated that the Neural Network approach can significantly improve the predictability of stock price performance. Y.L. Hsieh, Don-Lin Yang and Jungpin Wu [17] used data mining methods for sequential pattern mining. They prove that analysis of customer consumption behaviors and their buying habits can be judge with the help of data mining and it is helpful for the players of retail market. Md. Rafiul Hassan and
Baikunthu Nath [14] used Hidden Markov Models (HMM) approach to forecast financial market. Hidden Markov Models (HMM) is used to recognize pattern for the dynamic financial systems. Md. Rafiul Hassan and Baikunthu Nath develop hybrid system using artificial intelligence (AI) methods with HMM to increase accuracy in the forecasting financial market system. In the year 2008, Polat and Yildirim [15] introduced pattern recognition method and incorporate genetic algorithm and general regression neural network (GRNN) for the better results. Experimental results proves its efficiency by optimized general regression neural network (GRNN) and compare results with others also. Ching-Hseue cheng, TaiLiang chen, Liang-Ying Wei [3] uses hybrid forecasting model using multi-technical indicators to predict stock price trends. Authors described four indicators and uses correlation matrix within the data framework of financial market. Kuang $\mathrm{Yu}$ Huang, Chuen-Jiuan Jane [12] describes new methodologies with moving average autoregressive exogenous (ARX) prediction model. They combined moving average autoregressive exogenous (ARX) with grey system theory and rough set theory to create an automatic stock market forecasting and portfolio selection mechanism.

\section{LIMITATIONS OF NEURAL NETWORK BASED FORECASTING METHODS}

(i) Neural Network results are unstable. The neural network functions are Block Box function. The rules of operations are completely unknown.

(ii) Back propagation networks can be take long time to train the large amount of data.

(iii) Data mining techniques are used to archive the highest confidence up to $70 \%$ only.

(iv) Unlike a regression model, ARIMA model is not support the stationary time series data.

The limitations listed above by the survey work could be overcome by the proposed methodology of combined neural network and Hidden Markov model to improve the accuracy and effectiveness of the stock market price movements. These techniques are used to predict the hours based stock price movements, which may be deployed to week and month based stock price movements.

\section{GAP ANALYSIS OF EXISTING METHODOLOGIES AND MARKET DEMANDS}

From statistical to fractal methods, there is gap between existing techniques and demands of the market. In the one way, statistical methods are unable to suitably forecast market in a chaotic conditions and on the other hand chaotic market demands methods beyond fractal theory to predict the market. Neural network based methodologies are best suited for the market in chaotic conditions but developments till now is still need to improve. Our new method of forecasting will be based on the genetic algorithms and will try to fill the gap.

\section{CONCLUSIONS AND RESULTS}

This paper surveyed the Neural Network, Data mining, Neuro Fuzzy system and Markov Model in the area of stock market prediction. The NN and Markov model has ability to extract useful information from the data set so it is widely play very important role in stock market prediction. These approaches are used to control and monitor the entire the stock market price behavior and fluctuation. There are new approaches to 
known in-depth of an analysis of stock price variations. Markov Model can be used exclusively in the finance markets and forecasting of stock price. In this paper, we propose a forecasting method to provide better accuracy rather traditional methods. In future work neural network and Markov modal can also explore for other applications and comparative study with other time series analysis and forecasting models.

\section{REFERENCES}

[1] Abdulsalam sulaiman olaniyi, adewole, kayoed, Jimoh R.G, "Stock Trend Prediction using Regression Analysis - A Data Mining Approach”, AJSS journal, ISSN 22229833, 2010

[2] Akinwale Adio T, Arogundade O.T and Adekoya Adebayo F, "Translated Nigeria stock market price using artificial neural network for effective prediction", Journal of theoretical and Applied Information technology, 2009.

[3] Ching-Hsue cheng, Tai-Liang Chen, Liang-Ying Wei, "A hybrid model based on rough set theory and genetic algorithms for stock price forecasting", pp. $1610-1629$, 2010.

[4] Dase R.K. and Pawar D.D., "Application of Artificial Neural Network for stock market predictions: A review of literature" International Journal of Machine Intelligence, ISSN: 0975-2927, Volume 2, Issue 2, pp$14-17,2010$

[5] David Enke and Suraphan Thawornwong, "The use of data mining and neural networks for forecasting stock market returns, 2005.

[6] E. E. Peters, Fractal Market Analysis. Applying Chaos Theory to Investment \& Economics, Wiley, NewYork, 1994.

[7] Halbert White, "Economic prediction using neural networks: the case of IBM daily stock returns" Department of Economics University of California, San Diego, 2010.

[8] JingTao YAO and Chew Lim TAN, "Guidelines for Financial Prediction with Artificial neural networks", 2011

[9] K. Senthamarai Kannan, P. Sailapathi Sekar, M. Mohamed Sathik and P. Arumugam, "Financial stock market forecast using data mining Techniques", Proceedings of the international multiconference of engineers and computer scientists, 2010.

[10] Krishna Kumar Singh, Dr. Priti Dimri and Madhu Rawat, "Green Data Base for Stock Market: A case study of Indian Stock Market", IEEE Xplore digital library, pp. 848 - 853, September 2014, ISBN Number: 978-1-47994236-7, $25 \quad-26, \quad$ DOI: 10.1109/CONFLUENCE.2014.6949306.

[11] Krishna Kumar Singh, Dr. Priti Dimri and J. N. Singh, "Green Data Base Management System for intermediaries of the Indian stock market", IEEE Xplore digital library, pp. 1 - 5, March 2014, ISBN number: 978-1-4799-3063-0, 10.1109/CSIBIG.2014.7056996

[12] Kuang Yu Huang, Chuen-Jiuan Jane, "A hybrid model stock market forecasting and portfolio selection based on ARX, grey system and RS theories", Expert systems with Applications, pp. 5387 - 5392, 2009.

[13] M. Suresh babu, N.Geethanjali and B. Sathyanarayana "Forecasting of Indian Stock Market Index Using Data Mining \& Artificial Neural Nework", International journal of advance engineering \& application, 2011.

[14] Md. Rafiul Hassan and Baikunth Nath, "Stock Market forecasting using Hidden Markov Model: A New Approach", Proceeding of the $20055^{\text {th }}$ international conference on intelligent Systems Design and Application 0-7695 - 2286 -6/05, IEEE 2005.

[15] Ovunc Polat, Tulay Yıldırım. Genetic optimization of GRNN for pattern recognition without feature extraction, Expert Systems with Applications, vol. 34, pp: 2444 2448,2008

[16] Tiffany Hui-Kuang yu and Kun-Huang Huarng, "A Neural network-based fuzzy time series model to improve forecasting", Elsevier, pp: 3366-3372, 2010.

[17] Y. L. Hsieh, Don-Lin Yang and Jungpin Wu, "Using Data Mining to study Upstream and Downstream causa relationship in stock Market".

[18] Yi - Fan Wang, Shihmin Cheng and Mei-Hua Hsu, "Incorporating the Markov chain concepts into fuzzy stochastic prediction of stock indexes", Applied Soft Computing, pp. 613-617, 2010.

[19] Youngohc Yoon and George Swales, Proceedings of the IEEE International Conference on Neural networks, 156 $-162,1991$. 